\title{
Por los caminos de la Bioética en América Latina
}

Dr. Andrés Peralta Cornielle,

Director médico del Instituto Oncológico Regional del Cibao (República Dominicana) y miembro del Comité Internacional de Bioética de la UNESCO.

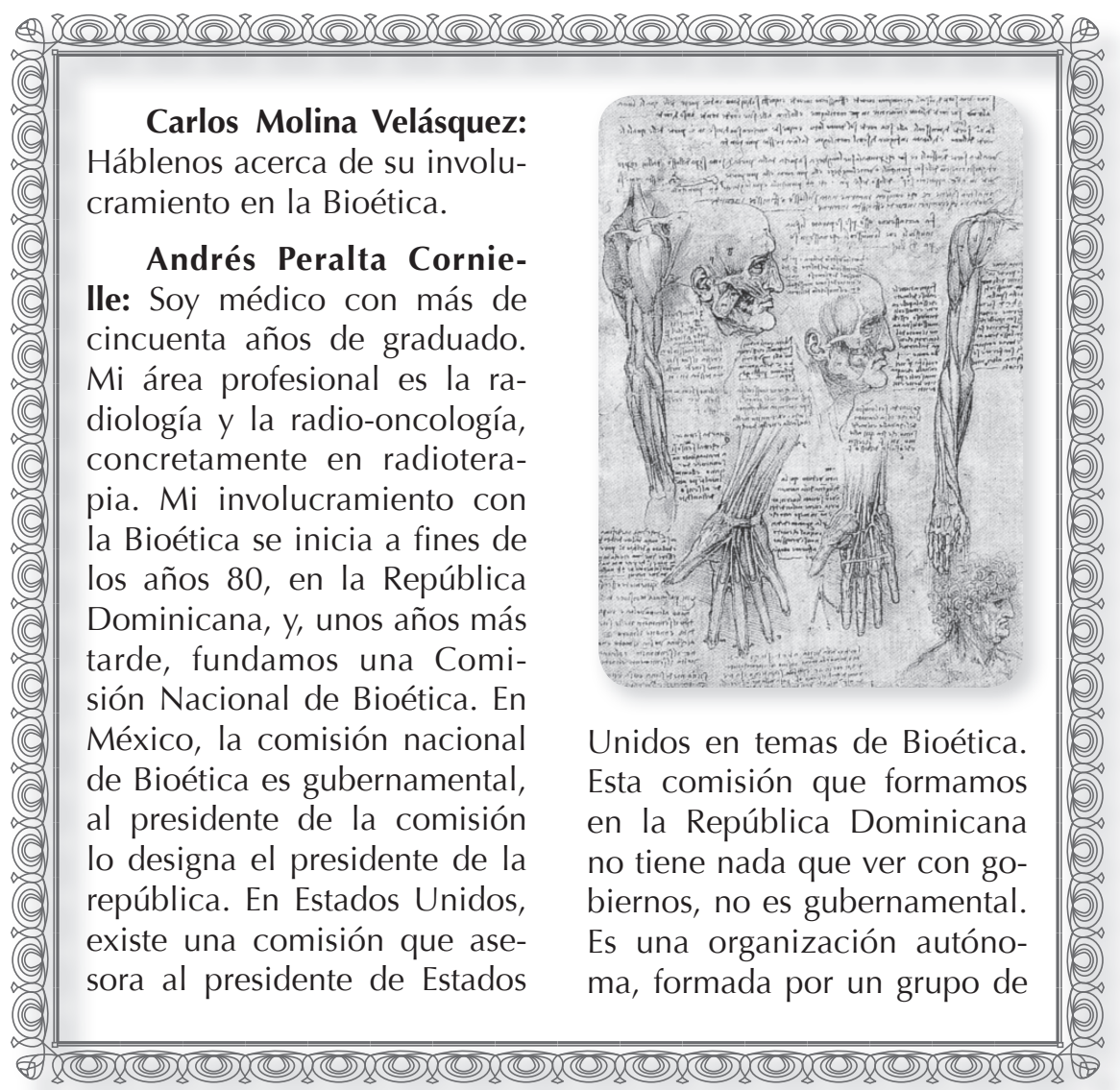


profesionales de diferentes áreas, no solo del área médica, sino del área del derecho, de la filosofía, de la tecnología, de la enfermería y en la que encontramos psicólogos, sociólogos... es multidisciplinaria, como debe ser la Bioética. De manera que mi involucramiento con la Bioética comienza en mi misma práctica profesional de la oncología, debido a que yo percibía que mis pacientes terminales demandaban algo que yo no estaba preparado a responder y esa fue la razón que me llevó hacia la Bioética.

Mi formación inicial en Bioética fue una formación autodidacta, hasta que decidí tener una formación académica. Esa formación académica comenzó con la Escuela Latinoamericana de Bioética, del Centro de Excelencia Oncológica (COE), en Gonnet (La Plata, Argentina), con el doctor José María Mainetti. Inicialmente, el director de esta escuela fue el doctor Juan Carlos Tealdi y nuestra Comisión Nacional de Bioética logró traer desde Argentina a Tealdi, el cual dictó un seminario de toda una semana. Y allí comenzó mi formación académica, junto a un grupo de profesionales de mi país. La segunda etapa de mi formación fue en la Universidad de Puerto Rico. Entre 1996 y 1998, asistí a unos seminarios de verano en la Universidad de Puerto Rico, seminarios de Bioética dirigidos a profesores de universidades norteamericanas, pero en los que me admitieron por la buena relación que yo mantenía con la Universidad de
Puerto Rico. Y en estos momentos me encuentro en la tercera etapa de mi formación académica, ya que acabo de terminar mi maestría en una universidad dominicana $y$, así como quienes están egresando de la maestría aquí en Costa Rica, yo me encuentro en el proceso de elaboración de la tesis.

CMV: ¿Sobre qué está preparando su tesis de maestría?

APC: Mi investigación es sobre educación en Bioética, precisamente. Estoy recabando información de las diferentes modalidades de la enseñanza de Bioética en América Latina, y concretamente en mi país, cuál ha sido el proceso que ha seguido. En la República Dominicana, la educación en Bioética tiene muchas limitaciones. Desde hace algunos años, algunas universidades con Escuela de Medicina han iniciado la enseñanza de Bioética a nivel de pregrado, y en el año 2000 se inició una maestría en Bioética en el Instituto Tecnológico de Santo Domingo. La razón por la que se creó esta maestría fue a consecuencia del programa de la unidad de Bioética de la Organización Panamericana de la Salud (OPS), el cual, durante cuatro años, estuvo en la Universidad de Chile, pero su objetivo inicial era que fuera estableciéndose en diferentes países de América Latina, que fuera una maestría itinerante. El director de esta maestría fue el doctor Diego Gracia, quien, después de dos promociones, salió de Chile y asentó la maestría en el Instituto Tecnológico 
de Santo Domingo. Ahí se formaron unas dos docenas de profesionales de América Latina, creo que hay egresados de Costa Rica. De hecho, el doctor Efrén Estrada, de El Salvador, se formó ahí. Después de que terminó el programa de maestría de la unidad de Bioética de la OPS, esta universidad estableció su propia maestría, de la cual yo soy un egresado.

Ahora bien, esta es la parte académica en mi formación. Pero aparte de esto, yo he tenido participación en el desarrollo de la Bioética en América Latina, a través de diferentes instituciones y organizaciones que han promovido la Bioética. La primera organización regional que se forma es la Federación Latinoamericana de Instituciones de Bioética (FELAIBE). Esta organización se funda en Colombia y Venezuela, y su primer congreso se celebra en São Paulo, Brasil. Su primer presidente fue el padre $\mathrm{Al}$ fonso Llano S.J., de la Universidad Javeriana (Colombia), fundador del Centro Nacional de Bioética (CENALBE). Posteriormente, la FELAIBE estuvo presidida por el maestro Velasco Suárez, de México; luego, el doctor Eduardo Sousa-Lennox, de Panamá, y actualmente la preside la doctora Marta Fracapani de Cuitiño, de Argentina. FELAIBE contribuyó grandemente a difundir la idea de la Bioética en América Latina. Hubo varios países que fueron sede del congreso: Argentina, México, Brasil, Colombia. Fue una gran labor la que hizo FELAIBE. Antes de FELAIBE, lo que había era la Escuela Latinoamericana de Bioética, del Centro de Excelencia Oncológica (COE), en Argentina. A mi juicio, su fundador, el doctor Mainetti, fue el promotor inicial de la Bioética en América Latina. Estoy hablando de la Bioética académica. Para mí, él es el padre de la Bioética en América Latina.

Luego surgen otras organizaciones, con las cuales yo también he estado involucrado, entre ellas el Foro Latinoamericano de Comités de Ética en Investigación en Salud (FLACEIS), que se funda en México, en el año 2000. Tuve el honor de ser vicepresidente, junto con Dafna Feinholz, quien presidió al FLACEIS del 2000 al 2005. Actualmente preside FLACEIS la doctora Dirce Guilhem, de Brasilia. FLACEIS también contribuyó, en el área de ética de la investigación en salud, a que varios países de América Latina, fundamentalmente de Centroamérica, desarrollaran o crearan comités de ética de la investigación en salud. Tuvimos aquí en Costa Rica un taller en el que participaron varios profesionales de todos los países de Centroamérica.

La otra organización de Bioética en la región, con la cual yo he estado involucrado, es la Red Latinoamericana y del Caribe de Bioética o Red Bioética, que es una organización fundada por la oficina regional de la UNESCO, que estaba establecida en México inicialmente. Su promotora fue la doctora Alya Saada, que era la representante de la UNESCO en la oficina regional 
en México. Estuve en el comité ejecutivo de la Red Bioética en los dos primeros años de su funcionamiento. A través de esta red celebramos varios eventos en Colombia, Uruguay, Brasil y México. Actualmente (2008) preside la Red el doctor Volnei Garrafa, de la Universidad de Brasilia. Yo permanezco todavía en la Red Bioética como miembro honorario. Fundamentalmente, la Red Bioética está realizando una excelente labor a través del programa de enseñanza, en los programas de enseñanza virtuales de Bioética clínica y otros cursos en Bioética de la investigación. Estos programas los dirige la profesora Susana Vidal, de Mendoza. Actualmente, la oficina regional de la UNESCO se trasladó de México a Montevideo y la doctora Vidal está provisionalmente a cargo de esto. El doctor Luis Justo, de Argentina, es uno de los profesores que imparte estos cursos virtuales de Bioética en la región.

Hay otras organizaciones de Bioética en América Latina, una de ellas es la Sociedad Internacional de Bioética (SIBI), que tiene un capítulo latinoamericano. Esto ha sido, en líneas generales, el desarrollo de la Bioética en América Latina.

CMV: Cuando se habla de Bioética, a muchos les suena como si se tratase de un discurso relacionado con problemas propios de los países del primer mundo. El profesor Volnei Garrafa hace una distinción que me parece muy sugerente, que usted conoce seguramente, y que me gustaría introducir en esta entre- vista: Él distingue entre problemas emergentes en la Bioética y problemas persistentes o reiterativos. ¿Cuál es su opinión al respecto?

APC: El doctor Volnei Garrafa y yo nos conocimos en el año 2000 y hemos coincidido en varias actividades sobre Bioética. Él es odontólogo, profesor de la Universidad de Brasilia. Además, la UNESCO tiene en varios países lo que se llama Cátedra UNESCO de Bioética; pues él es profesor titular de esa cátedra, en la Universidad de Brasilia. Yo he seguido el pensamiento del doctor Volnei Garrafa, el cual parte de la realidad de nuestros países latinoamericanos. Pudiste notar que el día de hoy escuchamos hablar del "Mantra de Georgetown", es decir, los cuatro principios básicos en Bioética: autonomía, beneficencia, no maleficencia y justicia. Entonces, Garrafa parte de la concepción de que en América Latina eso no es lo prioritario, ya que en nuestros países existen unos problemas persistentes a los que la Bioética, con esos cuatro principios, no puede hacer frente o buscar solución. Porque en América Latina hay "otros principios" donde la justicia esté por encima de la autonomía. En sus escritos, Garrafa promueve una ética de la intervención, una ética intervencionista. Los problemas de América Latina son fundamentalmente sociales, no individuales, y él aboga por una ética intervencionista que haga frente a los problemas persistentes en América Latina. Garrafa también habla de una Bioética fuerte, no de 
una Bioética académica de sentarse en un auditorio y hacer un bonito discurso, sino una Bioética fuerte, intervencionista, que se centre en la solución de los problemas sociales de nuestras comunidades.

Hay algo más: Yo soy miembro del Comité Internacional de Bioética de la UNESCO (IBC, en inglés). En la UNESCO, existe el área social que acoge la ética en las ciencias y dentro de esta área social de la UNESCO se crean dos organismos: el Comité Intergubernamental de Bioética y el Comité Internacional de Bioética. El primero está integrado por personas que designan los gobiernos miembros de la UNESCO, quienes no necesariamente son bioeticistas, sino que solamente representan los intereses de los gobiernos, que son los quienes los ubican. El segundo, el Comité Internacional de Bioética, tiene 34 miembros de muchos países, a título personal, y nos incorporamos a este organismo por invitación personal del director general de la UNESCO. Obviamente, el director busca evidencia de cuál ha sido la contribución de estos miembros a la Bioética en su desarrollo, en su discusión, en su implementación, en su promoción y yo tuve el honor de que, en el 2005, se me invitara a incorporarme a dicho comité. Es una designación por cuatro años, así que mi membresía concluye en el 2009.

El Comité Internacional de Bioética de la UNESCO seleccionó dos temas para trabajarlos en profundidad. Un tema se Ilama "Responsabilidad social en salud" y el otro tema es "Consentimiento informado". Yo formé parte del segundo grupo, trabajamos durante dos años y produjimos un documento sobre consentimiento informado en diversos contextos clínicos. Pero como la UNESCO es un organismo multinacional, cualquier documento aprobado por la UNESCO debe tener el consenso de los países miembros para que el documento sea oficial. Por eso es que, una vez que en el Comité Internacional lo diseñamos, aprobamos, pulimos y entregamos la versión final, por mandato de los estatutos de la UNESCO el director general tiene que presentar el documento al Comité Intergubernamental, en donde intervienen los intereses políticos de los gobiernos. Ya esa reunión se produjo, el proyecto fue evaluado y los gobiernos hicieron una serie de sugerencias, a través de sus representantes. De manera que en el Comité Internacional tendremos que volver a retomar este documento este año y a pulirlo un poco más para que sea potable para la mayoría de los países. 